Notfall Rettungsmed $2021 \cdot 24: 271-273$ https://doi.org/10.1007/s10049-021-00882-0 Angenommen: 19. April 2021

Online publiziert: 2. Juni 2021

(c) Springer Medizin Verlag GmbH, ein Teil von Springer Nature 2021

\author{
N. Rott ${ }^{1,2} \cdot$ B. Dirks ${ }^{2} \cdot$ B. W. Böttiger ${ }^{1,2}$ \\ ${ }^{1}$ Klinik für Anästhesiologie und Operative Intensivmedizin, Universitätsklinikum Köln, Köln, Deutschland \\ ${ }^{2}$ Deutscher Rat für Wiederbelebung - German Resuscitation Council (GRC) e.V., c/o Sektion \\ Notfallmedizin, Universitätsklinikum Ulm, Ulm, Deutschland
}

\title{
Die neuen Reanimationsleitlinien 2021 in der deutschen Übersetzung - die BIG-FIVE- Überlebensstrategien gewinnen deutlich an Bedeutung
}

Zentrale Aussagen zur Durchführung einer Wiederbelebung und zur Postreanimationsbehandlung wurden 2021 weitgehend beibehalten. Gleichzeitig wurden viele Empfehlungen durch neue Literatur und aktuelle wissenschaftliche Evidenz noch besser begründet. Neu hinzugekommen sind die Kapitel „Epidemiologie“ und „Systeme, die Leben retten“. Dadurch rücken zentral für das Überleben wichtige Themen wie Laienreanimation, Telefonreanimation, Ersthelfersysteme (z. B. durch Apps), KIDS SAVE LIVES (die Schülerausbildung in Wiederbelebung) und Cardiac Arrest Zentren deutlich mehr in den Vordergrund.

Im Kapitel „Epidemiologie“stellen wir die aktuellen europäischen Zahlen zur Reanimation dar. Dabei zeigt sich, dass Deutschland im europäischen Vergleich mit einer Laienreanimationsquote von $40,2 \%$ - im Vergleich zu $58 \%$ im europäischen Durchschnitt und auch beim Überleben mit 11,2\% im Vergleich zu anderen europäischen Ländern, die bis zu $18 \%$ Überleben erreichen - gerade mal im Mittelfeld liegt $[1,2]$.

Das Kapitel „Systeme, die Leben retten“ zeigt auf, dass es ein ganzes System und ein Zusammenspiel vieler Faktoren braucht, um das Überleben bestmöglich zu verbessern [3].

Damit passen die neuen Leitlinien auch perfekt zu den im letzten Jahr veröffentlichten BIG-FIVE-Überlebensstrategien nach Kreislaufstillstand, also fünf Strategien, mit denen die größte
Verbesserung des Überlebens erreicht werden kann [4].

Die Erhöhung der Laienreanimationsquote - Punkt 1 der BIG FIVE [4], von der eine Verdreifachung des Überlebens erwartet wird, spielt im Kapitel „Systeme, die Leben retten" in den fünf Kernaussagen direkt eine doppelte Rolle. Hier wird gefordert, das Bewusstsein für Laienreanimation $\mathrm{zu}$ erhöhen, z.B. durch Beteiligung an Aktionstagen wie dem „World Restart a Heart Day“ (WRAH), sowie alle Schülerinnen und Schüler in Laienreanimation zu unterrichten (KIDS SAVE LIVES). Während die Beteiligung am WRAH in Deutschland sehr intensiv betrieben wird (selbst in Pandemiezeiten z. B. durch die Social-Media-Kampagne \#MySongCanSaveLives), besteht bei der Schülerausbildung noch erheblicher Handlungsbedarf. Obwohl seit 2014 eine Empfehlung des Schulausschusses der Kultusministerkonferenz existiert, fehlt es nahezu überall an einer flächendeckenden Umsetzung.

Von der Telefonreanimation - Punkt 2 der BIG FIVE [4] - wird eine Verdopplung des Überlebens erwartet, von Ersthelfersystemen - Punkt 3 der BIG FIVE [4] - eine 1,2- bis 2-fachen Verbesserung. Beide werden in den Kernaussagen des neuen Leitlinienkapitels gefordert. In Deutschland sind mehrere Ersthelfersysteme etabliert, doch eine flächendeckende Umsetzung fehlt hier genauso wie bei der Telefonreanimation (nur 23,4\% Telefonreanimation in 2019 [2]). 
Punkt 4 der BIG FIVE [4] ist eine gute und flächendeckende Advanced-LifeSupport(ALS)-Versorgung, von der eine Verdopplung des Überlebens erwartet wird. Hierzu zählen u.a. gute Notarztsysteme, wie sie bei uns flächendeckend umgesetzt sind.

Punkt 5 [4] sind sogenannte Cardiac Arrest Zentren, also spezialisierte Kliniken zur Versorgung von Patientinnen und Patienten nach präklinischem Kreislauf-Stillstand. Von ihnen wird eine Verdopplung des Überlebens erwartet, deshalb werden sie in den Kernaussagen des Leitlinienkapitels ebenfalls gefordert [3]. Kriterien für Cardiac Arrest Zentren sind in Deutschland seit 2017 vorhanden, Zertifizierungsaudits werden seit Ende 2018 durchgeführt und die Zahl der zertifizierten Zentren wächst stetig an $[5,6]$.

Die BIG-FIVE-Überlebensstrategien - und dabei insbesondere die ersten Glieder der Überlebenskette, damit Patientinnen und Patienten die Klinik erreichen - gewinnen zunehmend und weiter an Bedeutung. Die Politik ist gefordert, eine flächendecke Umsetzung der hocheffektiven und das Überleben verbessernden Maßnahmen auch in Deutschland bzw. im deutschsprachigen Raum voranzutreiben.

Die Kapitel der neuen Leitlinien behandeln folgende Themen:

- Epidemiologie [1]

- Systeme, die Leben retten [3]

- Basismaßnahmen zur Wiederbelebung Erwachsener (Basic Life Support [BLS]) [7]

- Erweiterte Reanimationsmaßnahmen für Erwachsene (Advanced Life Support [ALS]) [8]

- Kreislaufstillstand unter besonderen Umständen [9]

- Postreanimationsbehandlung [10]

- Erste Hilfe [11]

- Versorgung und Reanimation des Neugeborenen nach der Geburt (Newborn Life Support [NLS] [12]

- Lebensrettende Maßnahmen bei Kindern (Pediatric Life Support [PLS]) [13]

- Ethik [14]

- Ausbildung [15]

Eine Kurzversion „Leitlinien kompakt“ in deutscher Sprache war bereits zeit- gleich zur Veröffentlichung der englischsprachigen Leitlinien (https://www. cprguidelines.eu/) auf der Website des GRC $\mathrm{zu}$ finden und ist dort weiterhin kostenfrei für alle abrufbar (https://www. grc-org.de/wissenschaft/leitlinien).

Wir danken allen Übersetzerinnen und Übersetzern, die diese zeitnahe Publikation der deutschsprachigen Leitlinien ermöglicht haben, sehr herzlich und wünschen Ihnen nun viel Vergnügen bei der Lektüre und natürlich sehr viel Erfolg bei Ihrer klinischen Patientenversorgung auf aktuellstem Niveau.

Und bitte helfen Sie alle mit, die BIG FIVE auch auf politischer Ebene in unseren Ländern voranzubringen. Unser Ziel ist es, in naher Zukunft in Europa nicht mehr nur „Mittelmaß“ zu sein.

Es grüßen Sie sehr herzlich

Nadine Rott

Burkhard Dirks

Bernd Böttiger

Köln und Ulm, im April 2021

\section{Korrespondenzadresse}

\section{N. Rott}

Deutscher Rat für Wiederbelebung - German Resuscitation Council (GRC) e. V., c/o Sektion Notfallmedizin, Universitätsklinikum Ulm 89070 UIm, Deutschland rott@grc-org.de

Interessenkonflikt. B.W. Böttiger ist Schatzmeister und Interim und Immediate Past Director Science and Research des European Resuscitation Council (ERC); Vorstandsvorsitzender des Deutschen Rats für Wiederbelebung/German Resuscitation Council (GRC); Mitglied im Präsidium der Deutschen Interdisziplinären Vereinigung für Intensiv- und Notfallmedizin (DIVI); Gründer der Deutschen Stiftung Wiederbelebung, Associated Editor des European Journal of Anaesthesiology (EJA); Mitherausgeber der Zeitschrift Resuscitation; Schriftleiter der Zeitschrift Notfall + Rettungsmedizin, Mitherausgeber der Zeitschrift Brazilian Journal of Anesthesiology. Für Vorträge hat er Honorare der folgenden Firmen erhalten: Forum für medizinische Fortbildung (FomF), Baxalta Deutschland GmbH ZOLL Medical Deutschland GmbH, C. R. Bard GmbH, GS Elektromedizinische Geräte G. Stemple GmbH, Novartis Pharma GmbH, Philips GmbH Market DACH, Bioscience Valuation BSV GmbH. N. Rott ist Mitarbeiterin des GRC. B. Dirks ist Gründungsvorsitzender und Generalsekretär des Deutschen Rates für Wiederebelebung/German Resuscitation Council (GRC).

\section{Literatur}

1. Gräsner J-T, Herlitz J, Tjelmeland IBM et al (2021) Epidemiologie des Kreislaufstillstandes in Europa.
Leitlinien des European Resuscitation Council 2021. Notfall Rettungsmed. https://doi.org/10. 1007/s10049-021-00884-y

2. https://www.reanimationsregister.de/downloads/ oeffentliche-jahresberichte/rettungsdienst/168oeffentlicher-jahresbericht-2019/file.html. Zugegriffen: 12. Apr. 2021

3. Semeraro F, Greif R, Böttiger BW et al (2021) Lebensrettende Systeme Leitlinien des European Resuscitation Council 2021. Notfall Rettungsmed. https://doi.org/10.1007/s10049-021-00889-7

4. Böttiger BW, BeckerLB, Kern KB, LippertF, Lockey A, Ristagno G, Semeraro F, Wingen S (2020) BIG FIVE strategies for survival following out-of-hospital cardiac arrest. Eur J Anaesthesiol 37(11):955-958. https://doi.org/10.1097/EJA.0000000000001247

5. Rott N, Scholz KH, Busch HJ et al (2020) 50. Cardiac Arrest Center Audit - Zertifizierung erfolgreich etabliert. Notfall Rettungsmed 23:370. https://doi. org/10.1007/s10049-020-00761-0

6. Rott N, Scholz KH, Busch HJ, Frey N, Kelm M, Thiele H, Böttiger BW (2020) Cardiac arrest center certification for out-of-hospital cardiac arrest patients successfully established in Germany. Resuscitation 156:1-3. https://doi.org/10.1016/j. resuscitation.2020.08.007

7. Olasveengen TM, Semeraro F, Ristagno $G$ et al (2021) Basismaßnahmen zur Wiederbelebung Erwachsener („Basic Life Support" , „BBLL) Leitlinien des European Resuscitation Council 2021. Notfall Rettungsmed. https://doi.org/10.1007/s10049021-00885-x

8. Soar J, Böttiger BW, Carli P et al (2021) Erweiterte lebensrettende Maßnahmen für Erwachsene. Leitlinien des European Resuscitation Council 2021. Notfall Rettungsmed. https://doi.org/10. 1007/s10049-021-00893-x

9. Lott C, Truhlář A, Alfonzo A et al (2021) Kreislaufstillstand unter besonderen Umständen. Leitlinien des European Resuscitation Council 2021. Notfall Rettungsmed. https://doi.org/10.1007/s10049021-00891-z

10. Nolan JP, Sandroni C, Böttiger BW et al (2021) Postreanimationsbehandlung. Leitlinien des European Resuscitation Council und der European Society of Intensive Care Medicine 2021. Notfall Rettungsmed. https://doi.org/10.1007/s10049021-00892-y

11. Zideman DA, Singletary EM, Borra V et al (2021) Erste Hilfe. Leitlinien des European Resuscitation Council 2021. Notfall Rettungsmed. https://doi. org/10.1007/s10049-021-00886-w

12. Madara J, RoehrCC, Ainsworth Setal (2021) Versorgung und Reanimation des Neugeborenen nach der Geburt. Leitlinien des European Resuscitation Council 2021. Notfall Rettungsmed. https://doi. org/10.1007/s10049-021-00894-w

13. Van de Voorde P Patrick, Turner MN, Djakow J et al (2021) Lebensrettende Maßnahmen bei Kindern („Paediatric Life Support, PLS“). Leitlinien des European Resuscitation Council 2021. Notfall Rettungsmed. https://doi.org/10.1007/s10049021-00887-9

14. Mentzelopoulos SD, Couper K, Van de Voorde Petal (2021) Ethik der Reanimation und Entscheidungen am Lebensende. Leitlinien des European Resuscitation Council 2021. Notfall Rettungsmed. https:// doi.org/10.1007/s10049-021-00888-8

15. Greif R, Lockey A, Breckwoldt J et al (2021) Lehre in der Reanimation. Leitlinien des European Resuscitation Council 2021. Notfall Rettungsmed. https://doi.org/10.1007/s10049-021-00890-0 
Hier steht eine Anzeige.

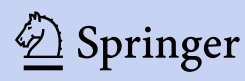

